\title{
On Solution to Traffic Flow Problem by Method of Characteristics
}

\author{
James, Torudonkumo and Eze, Everestus Obinwanne \\ Department of Mathematics and Statistics, Caritas University Amorji-Nike, Enugu state Nigeria.
}

\begin{abstract}
Our main purpose in this paper is to use the method of characteristics to solve traffic flow problems involving the conservation of cars. The method of characteristics is a technique of solving partial differential equations (PDEs) by imposing new coordinates, that is to say, change of coordinates. Note the traffic flow equation is classified as hyperbolic equation. We also discussed the relationship between flow rate, density and velocity.
\end{abstract}

Keywords: Traffic flow problem, Method of Characteristics, Flow rate, Density, Velocity, Change of Coordinates.

\section{Introduction}

The method of characteristics is a method that is used to solve initial value problems for general first order partial differential equations. The goal of the method of characteristics is to change coordinates, for example, from $(\mathrm{x}, \mathrm{t})$ to a new coordinate system $\left(\mathrm{x}_{\mathrm{o}}, \mathrm{s}\right)$ in which the partial differential equation becomes an ordinary differential equation along certain curves in the $\mathrm{x}, \mathrm{t}$ - plane, along the line $\mathrm{x}=0$. This can be done by constructing the characteristics for the equations over the region of known initial conditions and proceeding along these lines to determine the solutions for later times or for new regions.[Danielle L. Metcalf (2006)]

Billy M. Williams and Lester A. Hoel (2003) opined on the theoretical basis for modeling univariate traffic condition data streams as seasonal autoregressive integrated moving average processes. This foundation rests on the Wold decomposition theorem and on the assertion that a one-week lagged first seasonal difference applied to discrete interval traffic condition data will yield a weakly stationary transformation. Moreover, empirical results using actual intelligent transportation system data are presented and found to be consistent with the theoretical hypothesis.

Conclusions are given on the implications of these assertions and findings relative to ongoing intelligent transportation systems research, deployment, and operations.

More-over, the fundamental traffic flow law implies that $\mathrm{q}(\mathrm{x}, \mathrm{t})=\mathrm{p}(\mathrm{x}, \mathrm{t}) \mathrm{u}(\mathrm{x}, \mathrm{t})$ which is the relation of velocity, density, and flow rate (flux), an easy way to show this is to consider the number of cars that pass $\mathrm{x}=\mathrm{x}<0, \mathrm{x}>0$ in a very small time $\Delta \mathrm{t}$, that is, between $\mathrm{t} 0$ and $\mathrm{t} 0+\Delta \mathrm{t}$. [Richard Haberman (1998)]

Thus, few drivers would dispute the fact that congestion is caused by bottleneck, both recurrent and non-recurrent, and that the resulting queues can cause further problems if they become too long. Long queues can entrap cars do not wish to pass through the bottleneck that generated them, compounding the problem and causing spillovers. These can have widespread effects, such as "gridlock". [Daganzo F. Carlos (1999)]

In addition, from a practical point of view, it is important to have models that can predict reliably the things that matters, that is, bottleneck behavior and queue dynamics.

Nevertheless, traffic flow problem is a problem that we cannot avoid rather we find solution by developing mathematical model. [Richard Haberman] On some interval of roadway, between $x=a$ and $x=b$ which is bounded in this case but within this region we encounter "bumper to bumper" traffic which defines density at maximum, the number decreases due to cars leaving the region at $\mathrm{x}=\mathrm{b}$ and it implies maximum velocity (light turns green). In addition, cars slow down as the traffic density increases.

Note the relation between the velocity, density, and flow rate (flux) as stated earlier

i.e; $q=\rho u(\rho)$

where $\mathrm{q}=$ flow rate

$$
\begin{aligned}
& \rho=\text { density, and } \\
& \mathrm{u}=\text { velocity }
\end{aligned}
$$

then, the traffic flow equation of conservation of cars is given as

$\frac{\partial \rho}{\partial t}+\frac{d q}{d \rho} \cdot \frac{\partial \rho}{\partial x}=0$

must be solved using the initial condition $\mathrm{t}=0$

$\rho(x, 0)=\left\{\begin{array}{cl}\rho_{\max } & \text { if } x<0 \\ 0 & \text { if } x>0\end{array}\right.$ 
The equation (1.2) expresses a relationship between traffic flow derived by assuming that the numbers of cars are conserved, that is, cars are not created nor destroyed.

\section{Definition Of Some Concept}

$>$ Density-the number of cars per unit distance.

$>$ Flow-the number of cars per time unit that pass a given point on the highway.

Velocity- the rate of change in the position of an object as it moves in a particular direction.

$>$ Occupancy-the proportion of the road that is covered by cars.

\section{Methodology}

Our approach here is to use the Method of Characteristics to solve Traffic Flow problem (1.2) with an initial condition $\mathrm{t}=0$. The method of characteristics is a technique for solving partial differential equations. Typically, it applies to first order equations, although more generally the method is valid for any hyperbolic partial differential equation. Thus, the method is to reduce a partial differential equation to a family of ordinary differential equations along which the solution can be integrated from some initial data given on a suitable hyper surface.

\subsection{Conservation of Cars}

\section{Main Result/Discussion}

Suppose we have a highway of infinite length where the velocity and density are known; can we predict the pattern of traffic? First we consider $\rho(x, t)$ and $u(x, t)$ to be the two fundamental traffic variables. We have $\rho(\mathrm{x}, \mathrm{t})=$ traffic density, which is the number of cars at time $\mathrm{t}$ at position $\mathrm{x}$, and $\mathrm{u}(\mathrm{x}, \mathrm{t})=$ car velocity at position $\mathrm{x}$ and time $\mathrm{t}$, traffic flow $=\mathrm{q}(\mathrm{x}, \mathrm{t})$, which is the number of cars per hour passing position $\mathrm{x}$ at time $\mathrm{t}$, and thus $q(x, t)=\rho(x, t) u(x, t)$. Then the initial traffic density is $\rho(\mathrm{x}, 0)$, which is the traffic density at position $\mathrm{x}$ and time 0 , and the traffic velocity field for all time remains the same, $\mathrm{u}(\mathrm{x}, \mathrm{t})$. The motion of each car is determined by taking the derivative of position $\mathrm{x}$ with respect to $\mathrm{t}$, which satisfies the following first order differential equation:

$$
\frac{d x}{d t}=u(x, t) \text { with } x(0)=x_{0}
$$

Solving equation (4.1) determines the position of each car at future times. To be able to calculate the traffic density at future times we would need to know the traffic velocity and the initial density. We want to be able to calculate the density easily if we know the velocity. We choose an interval on any particular roadway between say $\mathrm{x}=\mathrm{a}$ and $\mathrm{x}=\mathrm{b}$, as illustrated below:

\begin{tabular}{|c|c|}
\hline & 1 \\
\hline$T$ & 1 \\
\hline 1 & 1 \\
\hline 1 & 1 \\
\hline $\begin{array}{l}1 \\
\text { a }\end{array}$ & I \\
\hline
\end{tabular}

Figure 1: cars entering and leaving a segment of roadway [1].

On this interval $[a, b]$, the number of cars, denoted $\mathrm{N}$, is the traffic density integrated:

$$
N(t)=\int_{x=a}^{x=b} \rho(x, t) d x
$$

Even with no exits or entrances on this roadway the number of cars on the interval between $\mathrm{x}=\mathrm{a}$ and $\mathrm{x}$ $=\mathrm{b}$ could still change in time. As cars enter at $\mathrm{x}=\mathrm{a}$, the number of cars increases and as cars leave $\mathrm{at} \mathrm{x}=\mathrm{b}$, the number of cars decreases, therefore the traffic flow q $(a, t)$ and $q(b, t)$ is not constant in time.

The rate of change of the number of cars with respect to time, $d N / d t$, is equal to the number of cars per unit time entering the interval $[a, b]$ at $x=a$ minus the number of cars per unit time exiting the interval $[a, b]$ at $x$ $=\mathrm{b}$, where the cars are always moving to the right, as illustrated in the equation below since the rate of change of the number of cars per unit time is the traffic flow at position a minus position $\mathrm{b}$ both at time $\mathrm{t}$ : 


$$
\frac{d N}{d t}=q(a, t)-q(b, t)
$$

Taking the derivative of both sides of equation (4.2) with respect to time gives the following:

$$
\frac{d N}{d t}=\frac{d}{d t} \int_{x=a}^{x=b} \rho(x, t) d x
$$

By combining equation (4.3) and equation (4.4), you get the result:

$$
\frac{d}{d t} \int_{x=a}^{x=b} \rho(x, t) d x=q(a, t)-q(b, t)
$$

And $q(a, t)-q(b, t)$ can be rewritten by taking the partial derivative of the right hand side of equation (4.5) with respect to $\mathrm{x}$, and then taking the integral from $\mathrm{x}=\mathrm{b}$ to $\mathrm{x}=\mathrm{a}$ gives the following equation:

$$
\frac{d}{d t} \int_{x=a}^{x=b} \rho(x, t) d x=\int_{x=b}^{x=a} \frac{\partial q(x, t)}{\partial x} d x
$$

To have the integral with the same interval, we need to use an integral property, which is to take the negative of the right hand side of equation (4.6):

$$
\frac{d}{d t} \int_{x=a}^{x=b} \rho(x, t) d x=-\int_{x=a}^{x=b} \frac{\partial q(x, t)}{\partial x} d x
$$

Moving the negative sign inside of the integral gives:

$$
\frac{d}{d t} \int_{x=a}^{x=b} \rho(x, t) d x=\int_{x=a}^{x=b}-\frac{\partial q(x, t)}{\partial x} d x
$$

We can now move the $d / d t$ inside of the integral to get the following equation; we can do this because derivatives and integrals are interchangeable. If you move the derivative inside the integral and it has a function of two variables, then the derivative becomes a partial derivative:

Equation (4.9) implies:

$$
\int_{x=a}^{x=b} \frac{\partial}{\partial t} \rho(x, t) d x=\int_{x=a}^{x=b}-\frac{\partial q(x, t)}{\partial x} d x
$$

$$
\int_{x=a}^{x=b}\left[\frac{\partial}{\partial t} \rho(x, t)+\frac{\partial q(x, t)}{\partial x}\right] d x=0
$$

Equation (4.10) implies:

$$
\frac{\partial}{\partial t} \rho(x, t)+\frac{\partial q(x, t)}{\partial x}=0
$$

And from equation (4.11) we get

$$
\frac{d \rho(x, t)}{d t}+\frac{\partial q(x, t)}{\partial x}=0
$$

Suppressing equation (4.12), which is just not including the variables of the function, gives us:

$$
\frac{d \rho}{d t}+\frac{\partial q}{\partial x}=0
$$

This is the equation of conservation of cars.

We know from above that $q=\rho u$, and so therefore we can rewrite the $\partial q / \partial x$ as the following: 


$$
\frac{\partial q}{\partial x}=\frac{\partial}{\partial x} q(\rho, u) .
$$

Which implies

$$
\frac{\partial q}{\partial x}=\frac{\partial q}{\partial \rho} \frac{\partial \rho}{\partial x}+\frac{\partial q}{\partial u} \frac{\partial u}{\partial x}
$$

Now combining equation (4.13) with equation (4.15) we get the following:

$$
\frac{\partial \rho}{\partial t}+\frac{\partial q}{\partial \rho} \frac{\partial \rho}{\partial x}+\frac{\partial q}{\partial u} \frac{\partial u}{\partial x}=0
$$

Which is still the conservation of cars since equation (4.16) is the same as equation (4.13).

Now, assume that $\boldsymbol{u}=\boldsymbol{u}(\rho)$.Taking the derivative to the velocity with respect to the position, $\mathrm{x}$ gives:

$$
\frac{\partial u}{\partial x}=0
$$

Combining equation (4.16) and equation (4.17) we get the following result:

$$
\frac{\partial \rho}{\partial t}+\frac{\partial q}{\partial \rho} \frac{\partial \rho}{\partial x}=0
$$

\subsection{A Velocity-Density Relationship}

There are many factors that have an effect on the speed at which a car can go since it is operated by an individual. The person operating one car may want to drive faster than another person in a different vehicle. Once the traffic becomes a lot heavier, however, lane changing and speed are at a minimum for every driver on the road since it is difficult to change lanes when there are more vehicles on the road and it is not always possible to go the speed you want when there are more vehicles on the road. A lot of times you get stuck going the same speed at which the flow of traffic is moving.

With all of these types of observations, we can make a simplifying assumption that at any point along the road the velocity of a car only depends on the density of cars. This is illustrated in the equation below, which was mentioned above in the explanation of the conservation of cars:

$$
u=u(\rho)
$$

As mentioned above, cars velocity can be at a maximum when there are very little to no cars at all on the road with them. So when there are no other cars at all on the road, this means that the density is at zero, and therefore the velocity will be at a maximum as illustrated with equation (4.17) with the density at zero below:

$$
u(0)=u_{\max }
$$

As more and more cars per mile that join the road way their presence will slow down the car, and as the density increases more, the velocity of the cars would continue to decrease. Thus the rate of change, which is the derivative of the velocity with respect to density, is defined as below:

$$
\frac{d u}{d \rho} \equiv u^{\prime}(\rho) \leq 0
$$

Once density is at a maximum, then cars will move at zero velocity, or stand still:

$$
u\left(\rho_{\max }\right)=0
$$

Therefore the car velocity vs. the traffic density is steady decreasing.

\subsection{Elementary Traffic Model}

As shown above, in general the car velocity is a decreasing function of density. At zero density, cars move the fastest which was denoted $u_{\max }$ and the maximum density was denoted $\rho_{\max }$ where the car velocity is zero. The simplest relationship to satisfy these properties is let: 


$$
u(\rho)=u_{\max }\left(1-\frac{\rho}{\rho_{\max }}\right)
$$

in which from the fact $q=\rho u$ the flow is given by

$$
q(\rho)=u_{\max } \rho\left(1-\frac{\rho}{\rho_{\max }}\right)=u_{\max }\left(\rho-\frac{\rho^{2}}{\rho_{\max }}\right)
$$

And the density wave velocity satisfies

$$
c(\rho)=q^{\prime}(\rho)=u_{\max }\left(1-\frac{2 \rho}{\rho_{\max }}\right)
$$

[Haberman, R. 2003].

\subsection{Red Light Turning Green}

Now we assume the elementary model of traffic flow so that the traffic density satisfies

$$
\frac{\partial \rho}{\partial t}+u_{\max }\left(1-\frac{2 \rho}{\rho_{\max }}\right) \frac{\partial \rho}{\partial x}=0
$$

Behind a red light position is at zero, $\mathrm{x}=0$, which is traffic density at its maximum, while ahead of the light the traffic density is at zero. So at time $\mathrm{t}=0$, the initial conditions for when the light turns green are:

$$
\rho(x, 0)=\left\{\begin{array}{l}
\rho_{\text {max }}, x<0 \\
0, x>0
\end{array}\right.
$$

The characteristic velocity is

$$
\frac{d x}{d t}=u_{\max }\left(1-\frac{2 \rho}{\rho_{\max }}\right)
$$

The density is constant along the characteristics so that they satisfy

$$
x=u_{\max }\left(1-\frac{2 \rho}{\rho_{\max }}\right) t+x_{0}
$$

The characteristic velocity is $u_{\max }$ for $\rho=0$, while the characteristic velocity is $-u_{\max }$ for $\rho=\rho_{\max }$. And thus

$$
\rho(x, 0)=\left\{\begin{array}{l}
\rho_{\text {max }}, x<-u_{\max } t \\
0, x>u_{\text {max }} t
\end{array}\right.
$$

The information that the traffic light turns green spreads backward at density velocity $-u_{\max }$. This is why each car has to wait until the car in front of them moves before they themselves can move. The characteristics are illustrated below: 


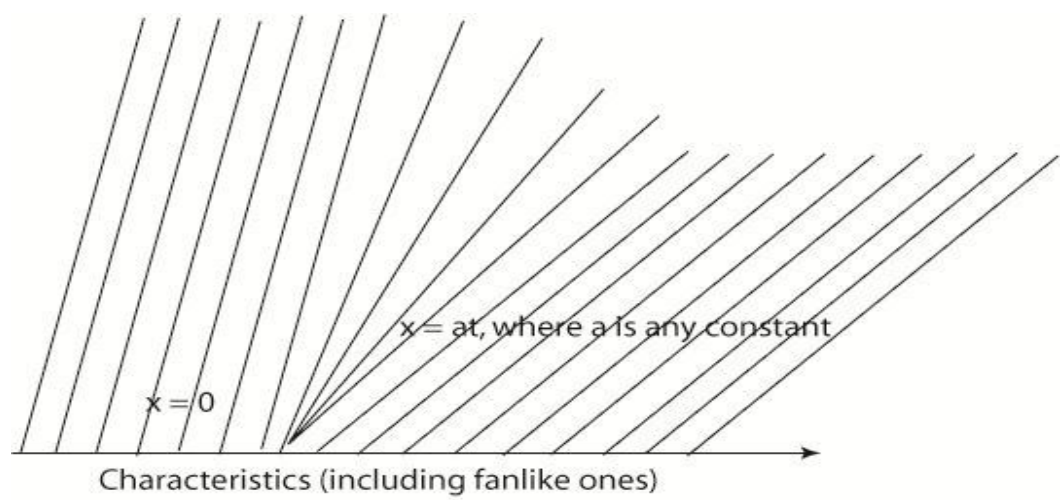

Figure 2 [Haberman, R. 1998]

Fanlike characteristics are where they generate out from the origin, $\mathrm{x}=0$.

To obtain the density at other points, we note that the family of fanlike characteristics that all start at $x_{0}=0$. Thus in this region

$$
x=u_{\max }\left(1-\frac{2 \rho}{\rho_{\max }}\right) t
$$

Given $\mathrm{x}$ and $\mathrm{t}$ in this region, equation (4.29), we can solve for the density:

$$
\rho(x, t)=\frac{\rho_{\max }}{2}\left(1-\frac{x}{u_{\max } t}\right) \text { for }-u_{\max } t<x<u_{\max } t
$$

[Haberman, R. 2003].

\subsection{An Initial Value Problem:}

Suppose that traffic, which is moving uniformly along a single lane road, comes to the end of a line of traffic which is stopped at a red light as illustrated below:

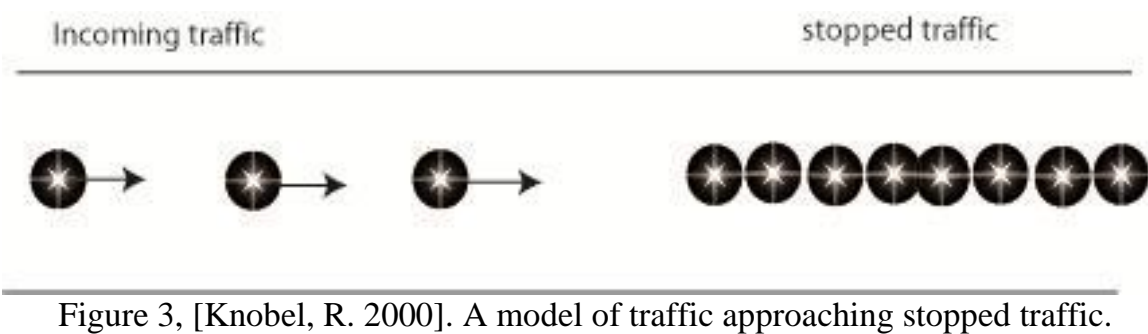

The cars that are already stopped are lined up with maximum density $\boldsymbol{u}_{1}$ cars per mile; while the approaching cars come to the end of the line have a uniform density $\boldsymbol{u}_{\mathbf{0}}$ cars per mile. Since $\boldsymbol{u}_{\mathbf{1}}$ is the maximum possible traffic density, the value of $u_{0}$ will satisfy $0<u_{0}<u_{1}$. From before, we know that $u(x, t)$ is the density, or cars per mile, of traffic at position $\mathrm{x}$ along the road at time $\mathrm{t}$. The fluctuation $\phi(x, t)$ represents the rate, cars per hour, that at which traffic passes by position $\mathrm{x}$ at time t. Letting $v_{1}$ denote maximum traffic velocity, the linear model for traffic velocity 


$$
v=v_{1}\left(1-\frac{u}{u_{1}}\right)
$$

results in the constitutive equation, the equation relating fluctuation and traffic density, is

$$
\phi=u v=v_{1}\left(u-\frac{u^{2}}{u_{1}}\right)
$$

Assuming that the road has no entrances or exits, the basic conservation law $u_{t}+\phi_{x}=f$ with fluctuation $\phi$ and source $f=0$ becomes

$$
u_{t}+v_{1}\left(1-\frac{2 u}{u_{1}}\right) u_{x}=0
$$

Let $x=0$ represent the location of the end of the stopped traffic at $t=0$. It is assumed that the stopped traffic extends indefinitely in one direction and the incoming traffic extends indefinitely in the other. In this case, the initial value problem

$$
u_{t}+v_{1}\left(1-\frac{2 u}{u_{1}}\right) u_{x}=0,-\infty<x<\infty, t>0
$$

Models the profile of traffic density $u(x, t)$ at later time t.

[Knobel, Roger (2000)]

\section{Conclusion}

So, we succeeded in obtaining the solution and therefore, we achieved our purpose. The method of characteristics is a very useful method of solving partial differential equations which was obtained by changing the coordinates from $(\mathrm{x}, \mathrm{t})$ to a new coordinate system $\left(\mathrm{x}_{\mathrm{o}}, \mathrm{s}\right)$ in which the partial differential equation becomes an ordinary differential equation with the help of imposing an initial condition $\mathrm{t}=0$. This work is also open to numerical approach to solve Traffic Flow Problem.

\section{References}

[1] Billy M. Williams, M.ASCE and Lester A. Hoel, F.ASCE (2003) Modeling and Forecasting Vehicular Trffic Flow as a Seasonal ARIMA Process: Theoretical Basis and Empirical Results. Journal of Transportation Engineering (C) ASCE/November/December 2003/665. DOI: 10.1061/(ASCE)0733-947X(2003/129:6(664).

[2] Daganzo, C.F. (1999). "The lagged cell-transmission model." Proc., $14^{\text {th }}$ Int. Symp. On Transportation and Traffic Theory, A. Ceder, ed., Pergamon, New York, 81-106.

[3] Danielle L. Metcalf (2006) Mathematics Descipline University of Minnesota, Morris, MN 56267.

[4] Dr. Scott A. Sarra. The Method of Characteristics with applications to Conservation Laws. October 17, 2002.

[5] Haberman, Richard. Mathematical Models, Mechanical Vibrations, Population Dynamics, and Traffic Flow. Philadelphia: Society of Industrial and Applied Mechanics, 1998. 275-382.

[6] Haberman, Richard. Applied Partial Differential Equations. Edition 4.

[7] Prentice Hall, 2003. 564-567.

[8] Hood, Alan. "Method of Characteristics." 30 Oct. 2000. 26 Mar. 2006 <http://www-solar.mes.stand.ac.uk/ alan/MT2003/PDE/node8.html>.

[9] Kampala Urban Traffic Improvement Plan (KUTIP), 2004. RITES Ltd., India, In association with Rank Consults (U) Ltd and Kagga and Partners Ltd., Uganda.

[10] Kendall, D.G., 1951. Some problems in the theory of queues. J. Royal Stat. Soc., Series B, 13:151-185.

[11] Knobel, Roger. An Introduction to the Mathematical Theory of Waves. Vol. 3. American Mathematical Society, 2000. 153-154.

[12] Wang, R. and H.J. Ruskin, 2003. Modeling traffic flow at single-lane urban roundabout. Comp. Phys. Commum., 147:570-576.

[13] Wilhelm, L., 1988. Introduction to the Theory of Traffic Flow. Berlin. 